\title{
Atorvastatin enhances interleukin-10 levels and improves cardiac function in rats after acute myocardial infarction
}

Christian Stumpf, MD; Sebastian Petzi; Katrin Seybold; Gerald Wasmeier, MD; Martin Arnold, MD; Dorette Raaz, MD; Atilla Yilmaz, MD; Werner G. Daniel, MD and Christoph D. Garlichs, MD

from the Dept. of Cardiology, University of Erlangen-Nuremberg, Erlangen, GERMANY

Short Title: Stumpf C., Atorvastatin and Heart Failure

Address correspondence to:

Christian Stumpf, MD

Dept. of Cardiology / Medical Clinic II

University of Erlangen-Nuremberg

Ulmenweg 18

91054 Erlangen, Germany

Phone: +4991318535000

Fax: +491212510790884

Email: ch.stumpf@web.de 


\section{A B S T R A C T}

Left ventricular (LV) remodeling is the basic mechanism of heart failure (HF) following myocardial infarction (MI). Whereas there is evidence that pro-inflammatory cytokines (including TNF- $\alpha$ and IL-6) are involved in the remodeling process, only little is known about the role of anti-inflammatory cytokines, such as IL-10. Since accumulating evidence revealed that statins possess anti-inflammatory properties, the goal of the present study was to elucidate the effect of atorvastatin on the modulation of the anti-inflammatory cytokine IL-10 and its effect on LV function in rats with HF subsequent to MI. Rats with MI, induced by permanent left anterior descending (LAD) branch ligation, were treated for 4 weeks with atorvastatin (10 mg/kg/d via oral gavage) starting on the first day after MI induction. Cardiac function was assessed by echocardiography and cardiac catheterization 4 weeks after MI induction. Membrane-bound and soluble fractions of TNF- $\alpha$, IL-6 and IL-10 protein, the ratio of TNF- $\alpha$ to IL-10, serum levels of MCP-1 as well as myocardial macrophage infiltration were analyzed. Treatment with atorvastatin significantly improved post MI LV function (FS $+120 \%$; $\mathrm{dP} / \mathrm{dt}_{\max }+147 \%$; LVEDP -27\%). Furthermore atorvastatin treatment markedly decreased levels of TNF- $\alpha$, IL-6 and MCP1 , reduced myocardial infiltration of macrophages and significantly increased myocardial and serum levels of the anti-inflammatory cytokine IL-10 thus shifting the balance between pro-inflammatory and anti-inflammatory cytokines towards the anti-inflammatory direction, as given in a significantly decreased TNF- $\alpha$ to IL-10 ratio,

Atorvastatin ameliorated early LV remodeling and improved LV function in rats with heart failure subsequent to MI. Our study suggests that the modulation of the balance between pro- and antiinflammatory cytokines towards the anti-inflammatory cytokine IL-10 is one salutary mechanism, of how atorvastatin influences post-MI remodeling and thus improves LV function.

Keywords: atrovastatin, interleukin-10, myocardial infarction, heart failure, inflammation 


\section{N TRODUCTION}

Chronic heart failure (CHF) is one of the major public health problems in the modern world. Despite major improvements in therapy over the last years the mortality rate in CHF patients has still remained at a very high level. Symptomatic heart failure continues to confer a worse prognosis than the majority of cancers in Europe and the United States, with a 1-year mortality averaging 45\%.[1] Myocardial infarction (MI) represents one of the most important causes for the development of cardiac failure.[2] Evidence has shown that pro-inflammatory cytokines such as tumor necrosis factor (TNF)- $\alpha$, interleukin (IL)-6 and monocyte chemoattractant protein (MCP)-1 are involved in the remodeling process following MI. Several studies furthermore suggest that the extent of cytokine production directly correlates with the severity of the disease process as well as the mortality rate [3-5] On the other hand, antiinflammatory cytokines such as IL-10 demonstrate potent anti-inflammatory properties and may neutralize the effects of pro-inflammatory cytokines.[6, 7] In this context, IL-10 has already been identified as a potentiaily important inhibitory mediator that may be involved in resolution of the post-infarction inflammatory reaction.[8]

In recent years accumulating evidence has revealed the anti-inflammatory effects of statins.[9-11] In numerous investigations it could be shown that administration of statins could decrease levels of CRP and pro-inflammatory cytokines with a concurrent decrease in cardiovascular events even in normocholesterolemic patients.[12, 13] However, the potential influence of statins on the anti-inflammatory cytokine IL-10 and its effect on cardiac function has not been clearly investigated and is still far from being understood.

Therefore, the aim of the present study was to elucidate the effect of atorvastatin on the modulation of the anti-inflammatory cytokine IL-10 and its effect on left ventricular function in rats with heart failure subsequent to MI. 


\section{METHODS}

\section{Animals}

Male Sprague-Dawley rats were maintained on standard rat chow and water ad libitum. Myocardial infarction was induced by occlusion of the left anterior descending coronary artery (LAD). Sham control animals were similarly handled, except the suture around the coronary artery was not tied. Body weight, general behaviour, and mortality of the animals were monitored on a regular basis. Cardiac function was assessed 4 weeks after induction of MI or sham operation. All animal study protocols were approved by the local authority for Laboratory Animal Care. The study conforms with the Guide for the Care and Use of Laboratory Animals published by the US National Institutes of Health (NIH Publication No. 8523, revised 1996).

\section{Myocardial Infarction Induction}

Myocardial infarction was induced in adult male Sprague-Dawley rats by ligating the LAD according to the technique by Pfeffer et al.:[14] The rats were anesthetized intraperitoneally with a mixture of xylazin $(1 \mathrm{mg} / 100 \mathrm{~g}$ bodyweight) and ketamin $(10 \mathrm{mg} / 100 \mathrm{~g}$ bodyweight), intubated with a fine polyethylene tube and put on positive-pressure ventilation (model 683, Harvard Apparatus, Inc., Southnatick, MA, USA). A left intercostal thoracotomy was performed to expose the heart, and the pericardium was opened. The LAD was looped by a single nylon suture (5.0) $1 \mathrm{~mm}$ from its origin, and gently tied. This procedure produced a clearly demarcated (cyanotic and bulged) area of acute ischemia corresponding to the distribution of the LAD distal to the occlusion, which results in MI of the free left ventricle and subsequently heart failure. After the ligature, the heart was repositioned in the chest, the skin was sutured, and air in the chest was removed with a syringe. After surgery, all rats were individually caged for a $24 \mathrm{~h}$ period of recovery. A sham-surgery group, in which all surgical procedures were the 
same, except that LAD ligation, was studied and did not present differences when compared to Control rats (data not included). After this period, all the infarcted rats recovered to good clinical condition, and exhibited no respiratory distress nor ascites, peripheral edema, or pleural effusion. A mortality about $25 \%$ among the animals submitted to LAD ligation was observed.

\section{Treatment of animals with atorvastatin}

Atorvastatin (Lipitor, Pfizer Inc.) was brought into suspension in phosphate-buffered saline (PBS). On the first day post-MI or sham operation, animals were randomized to receive either placebo (PBS) $(n=6)$ or atorvastatin ( $10 \mathrm{mg} / \mathrm{kg}, n=6$ ) given by oral gavage for 4 weeks. This dose was chosen because it turned out to be very effective in rats.[15-17]

\section{Echocardiography}

Echocardiographic examinations were performed under volatile isoflurane anaesthesia $(2.5 \%$ in oxygen, 500-700 ml/min; Draeger, Luebeck, Germany and Foehr Medical Instruments, Seeheim, Germany). Care was taken to maintain a stable, physiological heart rate at approximately $350 \mathrm{bpm}$ during the experiments monitored by a 3 channel ECG. Chests were shaved and rats were placed in left lateral decubitus position. For echocardiographic examination we used a Vivid 7 ultrasound system (General Electric Healthcare, USA) comprising a $10 \mathrm{MHz}$ transducer (General Electric, S10). All settings for pre- and postprocessing were adapted and optimised for small animals: Penetration depth was $2 \mathrm{~cm}$, near field was focussed, and gain was adjusted to optimal delineation. All recordings were stored digitally for subsequent off-line analysis.

Examinations were started in conventional two-dimensional echocardiography (2DE) with a frame rate of 80 per second resulting in 14 frames per heart cycle. The scan was performed in 
a parasternal short axis view to measure left ventricular diameter and endocardial areas in end-diastolic and end-systolic frames as recommended by the American Society of Echocardiography.[18] The time of end-diastole was therefore defined as time of maximum diameter of the LV in one heart cycle. Accordingly, end-systole was defined as the minimum diameter. Subsequently, we calculated the fractional shortening (FS).

\section{Hemodynamic measurements}

The left ventricular pressures were measured via a saline-filled cannula, which was inserted through the right carotid artery and connected to a pressure transducer. The cannula was inserted into the left ventricle to monitor left ventricular systolic (LVSP) and left ventricular end-diastolic pressure (LVEDP) as well as to measure maximum rate of rise of LV pressure $(\mathrm{dP} / \mathrm{d} t)$.

\section{Sample obtainment}

After completing the cardiac hemodynamic measurements, all the rats had their heart stopped in diastole by an intravenous injection of a $10 \%$ potassium chloride sodium $2 \sim 3 \mathrm{ml}$. The right ventricles were separated from the heart by the septums. The hearts were immediately frozen in liquid nitrogen and stored at $-70^{\circ} \mathrm{C}$.

\section{Myocardial TNF- $\alpha$ and IL-10 protein levels}

Hearts were washed with PBS. Viable ventricular tissue was flash frozen in liquid nitrogen. Frozen tissue $(0.5-1.0 \mathrm{~g})$ was homogenized, and membrane-bound and soluble fractions of TNF- $\alpha$ and $\mathrm{IL}-10$ proteins were collected and analyzed by ELISA using a commercially available kit (R \& D Systems, Minneapolis, MN, USA).[19, 20] Serum TNF- $\alpha$ and IL-10 were measured similarily. 


\section{Histological examination}

Hearts were stained with hematoxylin or subjected to immunostaining by using antibodies against CD68 (ED-1; Serotec). Immunoreactive materials were visualized by using a streptavidin-biotin staining kit. Macrophages (CD68-positive cells) were counted by a technician blinded to the treatment regimen. As negative controls, immunohistostaining was performed without the first antibodies.

\section{Statistical analysis}

All data are expressed as mean \pm SEM. For statistical analysis of the data, group means were compared by one-way ANOVA, and Bonferroni's test was used to identify differences between groups. Statistical significance was considered to be indicated by a value of $p<0.05$. For our sample size and for alpha 0.05 analysis revealed a power of 0.92 .

\section{RESULTS}

The mortality in the coronary artery-ligated groups, during or immediately after the surgery, was about $25 \%$. There was no significant difference in body weight gain between the coronary-artery ligated animals and their respective sham-operated controls. In the atorvastatin-treated MI group body weight was not different from the non-treated MI group.

\section{Cardiac function assessment}

Echocardiographic measurements and left heart catheterization were performed 4 weeks after surgery (Table 1). In the untreated group, MI resulted in a progressive increase in LV 
diameter which was slightly but not significantly reduced by atorvastatin. LV function in the MI group showed a progressive and significant impairment after MI as indicated by a decrease in FS that was prevented by treatment with atorvastatin (Table 1).

In the untreated MI group $\mathrm{dP} / \mathrm{dt}$ as an index of myocardial contractility as well as the LVSP were significantly reduced compared to the sham-operated group. LVEDP, on the other hand, was significantly increased. (Table 1, Fig. 1, Fig. 2) Treatment with atorvastatin significantly improved LVSP, dP/dt and reduced LVEDP (Fig. 2).

\section{Inflammatory and anti-inflammatory cytokines in postinfarct LV dysfunction}

4 weeks after coronary artery ligation levels of myocardial TNF- $\alpha$ as well as myocardial IL-6 significantly increased (Fig. 3 B,D). Systemically, serum levels of TNF- $\alpha$, IL-6 and MCP-1 were also found to be significantly increased in the untreated MI-group as compared to the Sham operated controls. (Fig. $3 \mathrm{~A}, \mathrm{C})$. On the other hand, the anti-inflammatory cytokine IL-10 was significantly decreased in its membrane-bound (myocardial) as well as soluble form 4 weeks after MI induction (Fig. 4). When comparing the inflammatory to the anti-inflammatory 'profile' in the MI group, the ratio of TNF- $\alpha$ to IL-10 was found to be significantly higher in the MI group (Fig. 5).

4-week-treatment with atorvastatin significantly decreased myocardial and serum levels of TNF- $\alpha$ as well as IL-6 compared to the untreated MI group (Fig. 3). A similar effect was seen for the CC chemokine MCP-1, which was significantly decreased under therapy with atorvastatin (Fig. $6 \mathrm{~A})$.

On the other hand atorvastatin significantly increased myocardial and serum levels of the antii-inflammatory cytokine IL-10 (Fig. 4).

Atorvastatin treatment shifted the balance between pro-inflammatory (TNF- $\alpha$ ) and antiinflammatory cytokines (IL-10) towards the anti-inflammatory IL-10 as seen in a significantly decreased TNF- $\alpha$ to IL-10 ratio (Fig. 5). 


\section{Infiltration of macrophages}

Macrophages are key regulators of the early LV remodeling process after MI. To determine the rate of macrophage infiltration post MI, the number of macrophages infiltrated into the infarct zone was quantified (Fig ๑). Myocardium of non-treated MI rats had significantly stronger infiltration of macrophages as compared to atorvastatin-treated MI rats (Fig. 6 B).

\section{DISCUSSION}

Atorvastatin, when started within 24 hours after MI, markedly ameliorates LV systolic function. This effect is accompanied not only by the reduction of myocardial as well as serum levels of pro-inflammatory cytokines (including TNF- $\alpha$, IL-6) and chemokines (MCP-1), but also by a reduction in myocardial macrophage infiltration and additionally by an increase of myocardial and serum levels of the anti-inflammatory cytokine IL-10.

Several studies provided evidence that the extent of cytokine production directly correlates with the severity of HF.[3, 4] In our study, we could find a significant increase in myocardial and serum levels of the pro-inflammatory cytokines TNF- $\alpha$ and IL-6 as well as an increase of the CC chemokine MCP-1 at 4 weeks post MI. On the other hand, the levels of the antiinflammatory cytokine IL-10 showed a significant reduction in its membrane-bound as well as its soluble form. Interestingly, this reduction also significantly correlated with depressed cardiac function. As an anti-inflammatory cytokine, IL-10 is already known to inhibit the production of pro-inflammatory cytokines (including TNF- $\alpha$, IL-6) thus suppressing the inflammatory response.[21, 22] Recently, it could be demonstrated that reperfused infarction in IL-10-deficient mice is associated with a $75 \%$ mortality, whereas no death occurred in wildtype animals.[23] Furthermore, a direct correlation between myocardial IL-10 mRNA and HF 
with time has also been reported.[24] In a recent study we could show that patients with advanced congestive HF (NYHA class III and IV) had significantly decreased levels of serum IL-10 and a significantly increased TNF- $\alpha$ to IL-10 ratio.[25]

Since in the past decade more and more studies have revealed the anti-inflammatory effects of statins, statins have been tested in experimental settings beyond their well-known cholesterol-lowering properties.[26, 27] Bauersachs et al. reported that cerivastatin improved LV remodeling in rats after a large MI.[28] Tiefenbacher et al demonstrated that fluvastatin can significantly reduce MI size in a reperfusion model.[29] In our study, in the MI model of permanent coronary artery occlusion, treatment with atorvastatin markedly ameliorated LV remodeling and LV function, supported by an increased FS, $\mathrm{dP} / \mathrm{dt}_{\max }$ and decreased LVEDP. Treatment with atorvastatin furthermore not only reduced levels of TNF- $\alpha$, IL- 6 and MCP-1, but also increased myocardial and serum levels of $\mathrm{IL}-10$, thus shifting the balance between pro-inflammatory and anti-inflammatory cytokines towards the anti-inflammatory direction.

Even though, statins have already shown to upregulate IL-10 levels in experimental encephalomyelitis[30], rheumatologic therapy[31] and after cardiac surgery[32], their influence on IL-10 and thus on post MI LV function has not been clearly investigated. Our data are indicating that atorvastatin might improve LV remodeling in part through increasing IL-10 production and inhibiting expressions of TNF- $\alpha$, IL- 6 and MCP-1. Interestingly, animals treated with atorvastatin also show a significant reduction in myocardial infiltration of macrophages 4 weeks post-MI further suggesting its role in reduction of inflammation and thus influencing cardiac function. One possible explanation for the reduced macrophage infiltration could be the decrease in the levels of the CC chemokine MCP-1. However, the exact molecular mechanisms of the differential regulation of pro- and anti-inflammatory cytokines by atorvastatin are far from being clear. Ortego et al. reported that atorvastatin reduced NF- $\kappa \mathrm{B}$ activity, which preferentially promotes pro-inflammatory cytokine expression.[33] In another 
report our own group could show that statins downregulate CD40 expression, another important member of the TNF-receptor family with potent influence in chronic inflammatory disease states.[34] On the other hand upregulation of IL-10 by atorvastatin may be a potent mechanism in further downregulating pro-inflammatory cytokines (including TNF- $\alpha$, IL-6) and chemokines (MCP-1).[35-37] Even though there are many reports that atorvastatin can markedly decrease the production of pro-inflammatory cytokines and chemokines[10, 11], our study shows its effect on regulating the inflammatory balance towards the anti-inflammatory cytokine IL-10 and thus influencing post-MI LV remodeling and function in an animal model. Whereas there are clinical reports that confirm the beneficial effects of these experimental studies,[38, 39] the recently published CORONA study (Controlled Rosuvatin in Multinational Trial in Heart Failure), one of the first large, randomized explorations of a statin given explicitly for HF, could not show a significant effect on cardiovascular outcomes.[40] However this study showed a significantly reduced number of hospitalizations from CV causes and from HF. In our study, we could see a significant influence of atorvastatin on cardiac function, when starting the therapy within 24 hours post MI suggesting that giving statins earlier in the natural history of HF might produce better results. Since there are reports that patients with low IL-10 levels might have a poorer cardiovascular prognosis, $[41,42]$ it is tempting to hypothesize that especially those patients could benefit from a therapy that is shifting the inflammatory balance towards the anti-inflammatory direction. However, further studies need to be done to shed light on some of these unresolved questions.

In summary, our study indicates that pro- and anti-inflammatory cytokines are important players in post-MI LV remodeling. The effect of atorvastatin on the modulation of the balance between pro- and anti-inflammatory cytokines towards the anti-inflammatory cytokine IL-10 is one salutary mechanism of how atorvastatin influences post-MI remodeling and thus improves post-MI LV function. These data further support the concept that the balance between pro- 
and anti-inflammatory cytokines is a major determinant in the prognosis of post MI heart failure and therefore, enhancing anti-inflammatory cytokines may be a promising approach for medical treatment.

\section{ACKNOWLEDGEMENTS}

The study was supported by a grant of the Interdisciplinary Center for Clinical Research (IZKF) within the Medical Faculty of the University of Erlangen, Germany.

\section{DISCLOSURES}

None. 


\section{F I G U R E LE G E N D S}

\section{Figure 1:}

Echocardiographic images of rat hearts after sham-operation $(A)$ and induction of myocardial infarction (B). Top: 2-dimensional echocardiography at parasternal short-axis plane. Bottom: representative M-mode short-axis of left ventricle (LV). ESD, end-systolic dimensions; EDD, end-diastolic dimensions; RV, right ventricle.

\section{Figure 2:}

(A) Left ventricular enddiastolic pressure (LVEDP) and (B) $\mathrm{dP} / \mathrm{dt}_{\max }$, parameter of systolic function, in sham-operated rats and rats with myocardial infarction (MI), untreated and treated with atorvastatin for 4 weeks. Data are expressed as mean \pm SEM. $* p<0.01$ vs sham, $+p<0.05$ atorvastatin treatment vs untreated MI group.

\section{Figure 3:}

Soluble (A) and (B) myocardial membrane-bound IL-6 as well as soluble (C) and myocardial membrane-bound (D) TNF- $\alpha$ protein concentration in sham-operated rats and rats with myocardial infarction (MI), untreated and treated with atorvastatin for 4 weeks.

Data are expressed as mean $\pm \mathrm{SEM}$. $* p<0.001$ vs sham rats; $\dagger p<0.01$ vs. MI rats._

\section{Figure 4:}

Myocardial membrane-bound (A) and soluble (B) IL-10 concentration in sham-operated rats and rats with myocardial infarction (MI) untreated and treated with atorvastatin. Data are expressed as mean \pm SEM. $* p<0.001$ vs. sham rats; $\uparrow p<0.01$ vs. MI rats. 


\section{Figure 5:}

Ratio of soluble TNF- $\alpha$ to soluble IL-10 concentration in sham-operated rats and rats with myocardial infarction (MI) untreated and treated with atorvastatin. Data are expressed as mean \pm SEM. $* p<0.001$ vs. sham rats, $\dagger p<0.05$ vs. MI rats.

\section{Figure 6:}

(A) Serum MCP-1 levels and (B) myocardial infiltration of macrophages in sham-operated rats and rats with myocardial infarction (MI), untreated and treated with atorvastatin for 4 weeks. Data are expressed as mean \pm SEM. $* p<0.01$ vs sham rats; $\dagger p<0.01$ vs MI rats. 


\section{REFERENCES}

1. Cowie M. R., Wood D. A., Coats A. J. et al. (2000) Survival of patients with a new diagnosis of heart failure: a population based study. Heart. 83, 505-510.

2. Bolognese L., Cerisano G. (1999) Early predictors of left ventricular remodeling after acute myocardial infarction. Am Heart J. 138, S79-83.

3. Dibbs Z., Kurrelmeyer K., Kalra D. et al. (1999) Cytokines in heart failure: pathogenetic mechanisms and potential treatment. Proc Assoc Am Physicians. 111, 423-428.

4. Mann D. L. (1999) Inflammatory mediators in heart failure: homogeneity through heterogeneity. Lancet. 353, 1812-1813.

5. Torre-Amione G., Kapadia S., Benedict C., Oral H., Young J. B., Mann D. L. (1996) Proinflammatory cytokine levels in patients with depressed left ventricular ejection fraction: a report from the Studies of Left Ventricular Dysfunction (SOLVD). J Am Coll Cardiol. 27, 1201-1206.

6. Ono K., Matsumori A., Shioi T., Furukawa Y., Sasayama S. (1998) Cytokine gene expression after myocardial infarction in rat hearts: possible implication in left ventricular remodeling. Circulation. 98, 149-156.

7. Yamaoka M., Yamaguchi S., Okuyama M., Tomoike H. (1999) Anti-inflammatory cytokine profile in human heart failure: behavior of interleukin-10 in association with tumor necrosis factor-alpha. Jpn Circ J. 63, 951-956.

8. Frangogiannis N. G., Mendoza L. H., Lindsey M. L. et al. (2000) IL-10 is induced in the reperfused myocardium and may modulate the reaction to injury. J Immunol. 165, 2798-2808.

9. Node K., Fujita M., Kitakaze M., Hori M., Liao J. K. (2003) Short-term statin therapy improves cardiac function and symptoms in patients with idiopathic dilated cardiomyopathy. Circulation. 108, 839-843. 
10. Rezaie-Majd A., Maca T., Bucek R. A. et al. (2002) Simvastatin reduces expression of cytokines interleukin-6, interleukin-8, and monocyte chemoattractant protein-1 in circulating monocytes from hypercholesterolemic patients. Arterioscler Thromb Vasc Biol. 22, 1194-1199.

11. Inoue I., Goto S., Mizotani K. et al. (2000) Lipophilic HMG-CoA reductase inhibitor has an anti-inflammatory effect: reduction of MRNA levels for interleukin-1beta, interleukin6, cyclooxygenase-2, and p22phox by regulation of peroxisome proliferator-activated receptor alpha (PPARalpha) in primary endothelial cells. Life Sci. 67, 863-876.

12. Colhoun H. M., Betteridge D. J., Durrington P. N. et al. (2004) Primary prevention of cardiovascular disease with atorvastatin in type 2 diabetes in the Collaborative Atorvastatin Diabetes Study (CARDS): multicentre randomised placebo-controlled trial. Lancet. 364, 685-696.

13. Albert M. A., Danielson E., Rifai N., Ridker P. M. (2001) Effect of statin therapy on Creactive protein levels: the pravastatin inflammation/CRP evaluation (PRINCE): a randomized trial and cohort study. Jama. 286, 64-70.

14. Pfeffer M. A., Pfeffer J. M., Fishbein M. C. et al. (1979) Myocardial infarct size and ventricular function in rats. Circ Res. 44, 503-512.

15. Birnbaum Y., Lin Y., Ye Y., Merla R., Perez-Polo J. R., Uretsky B. F. (2008) Pretreatment with high-dose statin, but not low-dose statin, ezetimibe, or the combination of low-dose statin and ezetimibe, limits infarct size in the rat. J Cardiovasc Pharmacol Ther. 13, 72-79.

16. Barsante M. M., Roffe E., Yokoro C. M. et al. (2005) Anti-inflammatory and analgesic effects of atorvastatin in a rat model of adjuvant-induced arthritis. Eur J Pharmacol. 516, 282-289.

17. Liantonio A., Giannuzzi V., Cippone V., Camerino G. M., Pierno S., Camerino D. C. (2007) Fluvastatin and atorvastatin affect calcium homeostasis of rat skeletal muscle fibers in vivo and in vitro by impairing the sarcoplasmic reticulum/mitochondria Ca2+release system. J Pharmacol Exp Ther. 321, 626-634. 
18. Cheitlin M. D., Armstrong W. F., Aurigemma G. P. et al. (2003) ACC/AHA/ASE 2003 Guideline Update for the Clinical Application of Echocardiography: summary article. A report of the American College of Cardiology/American Heart Association Task Force on Practice Guidelines (ACC/AHA/ASE Committee to Update the 1997 Guidelines for the Clinical Application of Echocardiography). J Am Soc Echocardiogr. 16, 1091-1110.

19. Torre-Amione G., Stetson S. J., Youker K. A. et al. (1999) Decreased expression of tumor necrosis factor-alpha in failing human myocardium after mechanical circulatory support : A potential mechanism for cardiac recovery. Circulation. 100, 1189-1193.

20. Baumgarten G., Kim S. C., Stapel H. et al. (2006) Myocardial injury modulates the innate immune system and changes myocardial sensitivity. Basic Res Cardiol. 101, 427-435.

21. de Vries J. E. (1995) Immunosuppressive and anti-inflammatory properties of interleukin 10. Ann Med. 27, 537-541.

22. Silvestre J. S., Mallat Z., Tamarat R., Duriez M., Tedgui A., Levy B. I. (2001) Regulation of matrix metalloproteinase activity in ischemic tissue by interleukin-10: role in ischemia-induced angiogenesis. Circ Res. 89, 259-264.

23. Yang Z., Zingarelli B., Szabo C. (2000) Crucial role of endogenous interleukin-10 production in myocardial ischemia/reperfusion injury. Circulation. 101, 1019-1026.

24. Ukimura A., Terasaki F., Fujioka S. et al. (2003) Quantitative analysis of cytokine mRNA expression in hearts from patients with nonischemic dilated cardiomyopathy (DCM). J Card Surg. 18 Suppl 2, S101-108.

25. Stumpf C., Lehner C., Yilmaz A., Daniel W. G., Garlichs C. D. (2003) Decrease of serum levels of the anti-inflammatory cytokine interleukin-10 in patients with advanced chronic heart failure. Clin Sci (Lond). 105, 45-50.

26. Undas A., Celinska-Lowenhoff M., Kaczor M., Musial J. (2004) New nonlipid effects of statins and their clinical relevance in cardiovascular disease. Thromb Haemost. 91, 1065-1077. 
27. Zhang J., Cheng X., Liao Y. H. et al. (2005) Simvastatin regulates myocardial cytokine expression and improves ventricular remodeling in rats after acute myocardial infarction. Cardiovasc Drugs Ther. 19, 13-21.

28. Bauersachs J., Galuppo P., Fraccarollo D., Christ M., Ertl G. (2001) Improvement of left ventricular remodeling and function by hydroxymethylglutaryl coenzyme a reductase inhibition with cerivastatin in rats with heart failure after myocardial infarction. Circulation. 104, 982-985.

29. Tiefenbacher C. P., Kapitza J., Dietz V., Lee C. H., Niroomand F. (2003) Reduction of myocardial infarct size by fluvastatin. Am J Physiol Heart Circ Physiol. 285, H59-64.

30. Stanislaus R., Gilg A. G., Singh A. K., Singh I. (2002) Immunomodulation of experimental autoimmune encephalomyelitis in the Lewis rats by Lovastatin. Neurosci Lett. 333, 167-170.

31. Arnaud C., Mach F. (2006) Potential antiinflammatory and immunomodulatory effects of statins in rheumatologic therapy. Arthritis Rheum. 54, 390-392.

32. Liakopoulos O. J., Dorge H., Schmitto J. D., Nagorsnik U., Grabedunkel J., Schoendube F. A. (2006) Effects of preoperative statin therapy on cytokines after cardiac surgery. Thorac Cardiovasc Surg. 54, 250-254.

33. Ortego M., Bustos C., Hernandez-Presa M. A. et al. (1999) Atorvastatin reduces NFkappaB activation and chemokine expression in vascular smooth muscle cells and mononuclear cells. Atherosclerosis. 147, 253-261.

34. Garlichs C. D., John S., Schmeisser A. et al. (2001) Upregulation of CD40 and CD40 ligand (CD154) in patients with moderate hypercholesterolemia. Circulation. 104, 2395-2400.

35. Bolger A. P., Sharma R., von Haehling S. et al. (2002) Effect of interleukin-10 on the production of tumor necrosis factor-alpha by peripheral blood mononuclear cells from patients with chronic heart failure. Am J Cardiol. 90, 384-389. 
36. Escofier N., Boichot E., Germain N., Silva P. M., Martins M. A., Lagente V. (1999) Effects of interleukin-10 and modulators of cyclic AMP formation on endotoxin-induced inflammation in rat lung. Fundam Clin Pharmacol. 13, 96-101.

37. de Waal Malefyt R., Abrams J., Bennett B., Figdor C. G., de Vries J. E. (1991) Interleukin 10(IL-10) inhibits cytokine synthesis by human monocytes: an autoregulatory role of IL-10 produced by monocytes. J Exp Med. 174, 1209-1220.

38. Sola S., Mir M. Q., Lerakis S., Tandon N., Khan B. V. (2006) Atorvastatin improves left ventricular systolic function and serum markers of inflammation in nonischemic heart failure. J Am Coll Cardiol. 47, 332-337.

39. Van Linthout S., Riad A., Dhayat N. et al. (2007) Anti-inflammatory effects of atorvastatin improve left ventricular function in experimental diabetic cardiomyopathy. Diabetologia. 50, 1977-1986.

40. Kjekshus J., Apetrei E., Barrios V. et al. (2007) Rosuvastatin in older patients with systolic heart failure. N Engl J Med. 357, 2248-2261.

41. Girndt M., Kaul H., Sester U. et al. (2002) Anti-inflammatory interleukin-10 genotype protects dialysis patients from cardiovascular events. Kidney Int. 62, 949-955.

42. Girndt M., Kohler H. (2003) Interleukin-10 (IL-10): an update on its relevance for cardiovascular risk. Nephrol Dial Transplant. 18, 1976-1979. 
TABLE 1. Hemodynamic and Echocardiographic Parameters

\begin{tabular}{|c|c|c|c|}
\hline & Sham & MI & + atorvastatin \\
\hline Rats, $n$ & 5 & & 6 \\
\hline Heart rate $[\mathrm{bpm}]$ & $390 \pm 32$ & 0 金24 & $408 \pm 31$ \\
\hline Infarct size $[\%]$ & & $48.8+\frac{+3}{7} .9$ & $46.3 \pm 5.2$ \\
\hline Left ventricular systolic pressure (LVSP) [mı & & 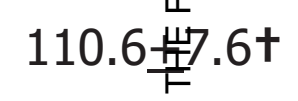 & $119.3 \pm 8.2$ \\
\hline Mean arterial pressure (MAP) $[\mathrm{mmHg}]$ & & $97.9 \underset{-2}{+6} .9$ & $99.8 \pm 7.6$ \\
\hline LV end-diastolic pressure (LVEDP) [mm & $4.5 \pm 1.9$ & $14.3 \pm \underline{\underline{4}} .9 *$ & $10.5 \pm 3.7 \S$ \\
\hline $\mathrm{dP} / \mathrm{d} t[\mathrm{mmHg} / \mathrm{s}]$ & $8953 \pm 423$ & $4425 \pm 520 *$ & $6532 \pm 478 \S$ \\
\hline LV end-diastolic parameter (LVEDD) [mm] & $5.3 \pm 0.8$ & $9.6 \pm 0.4 *$ & $8.2 \pm 0.6$ \\
\hline Fractional shortening (FS) [\%] & $40 \pm 2.24$ & $27.3 \pm 4.45 *$ & $32.9 \pm 3.72 \neq$ \\
\hline
\end{tabular}

Values are mean \pm SEM.

$* p<0.01,+p<0.05$ vs. sham rats, $\neq p<0.05, \S p<0.01$ vs. MI rats 

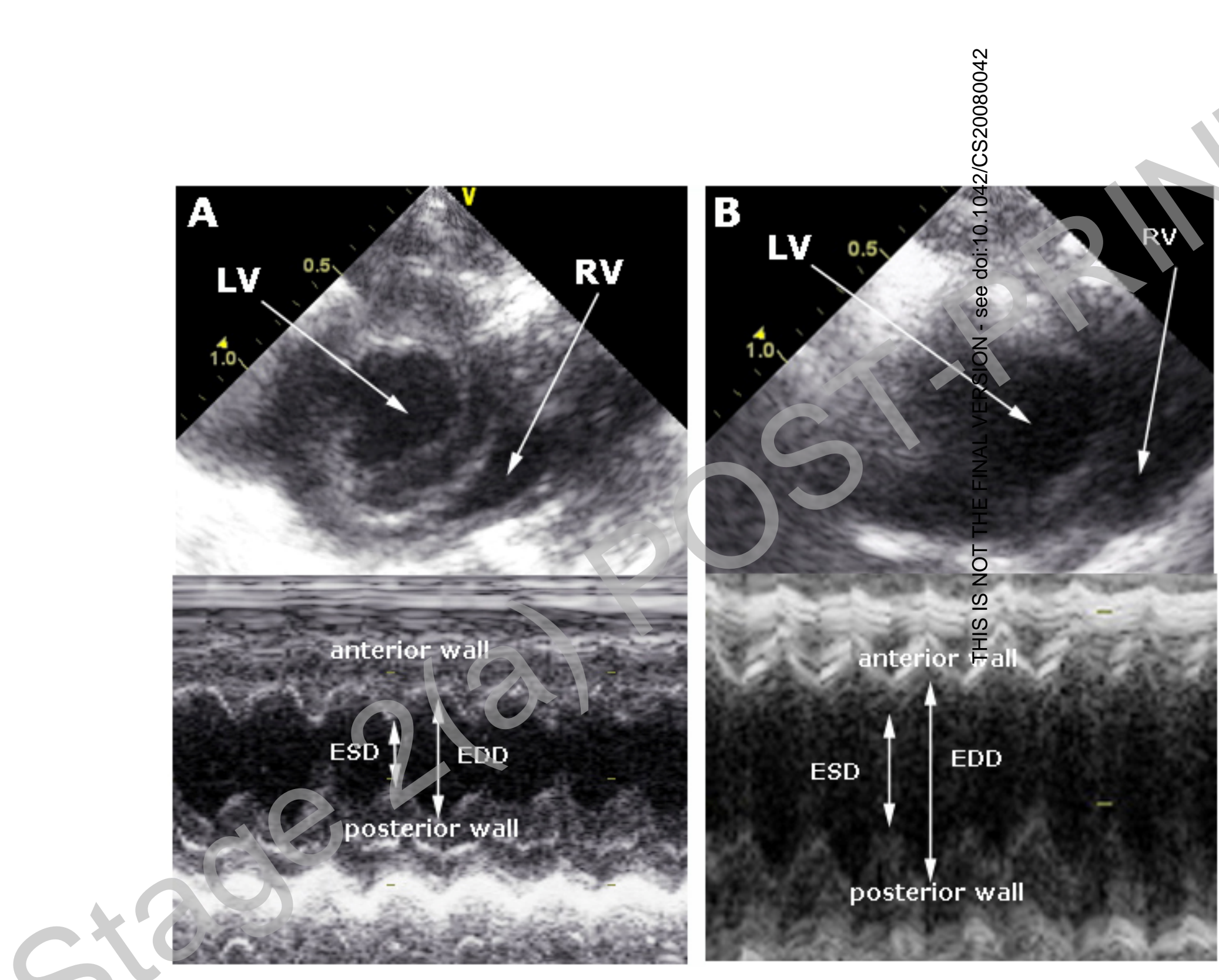

Figure 1

Licenced copy. Copying is not permitted, except with prior permission and as allowed by law. (c) 2008 The Authors Journal compilation (c) 2008 Biochemical Society 
A

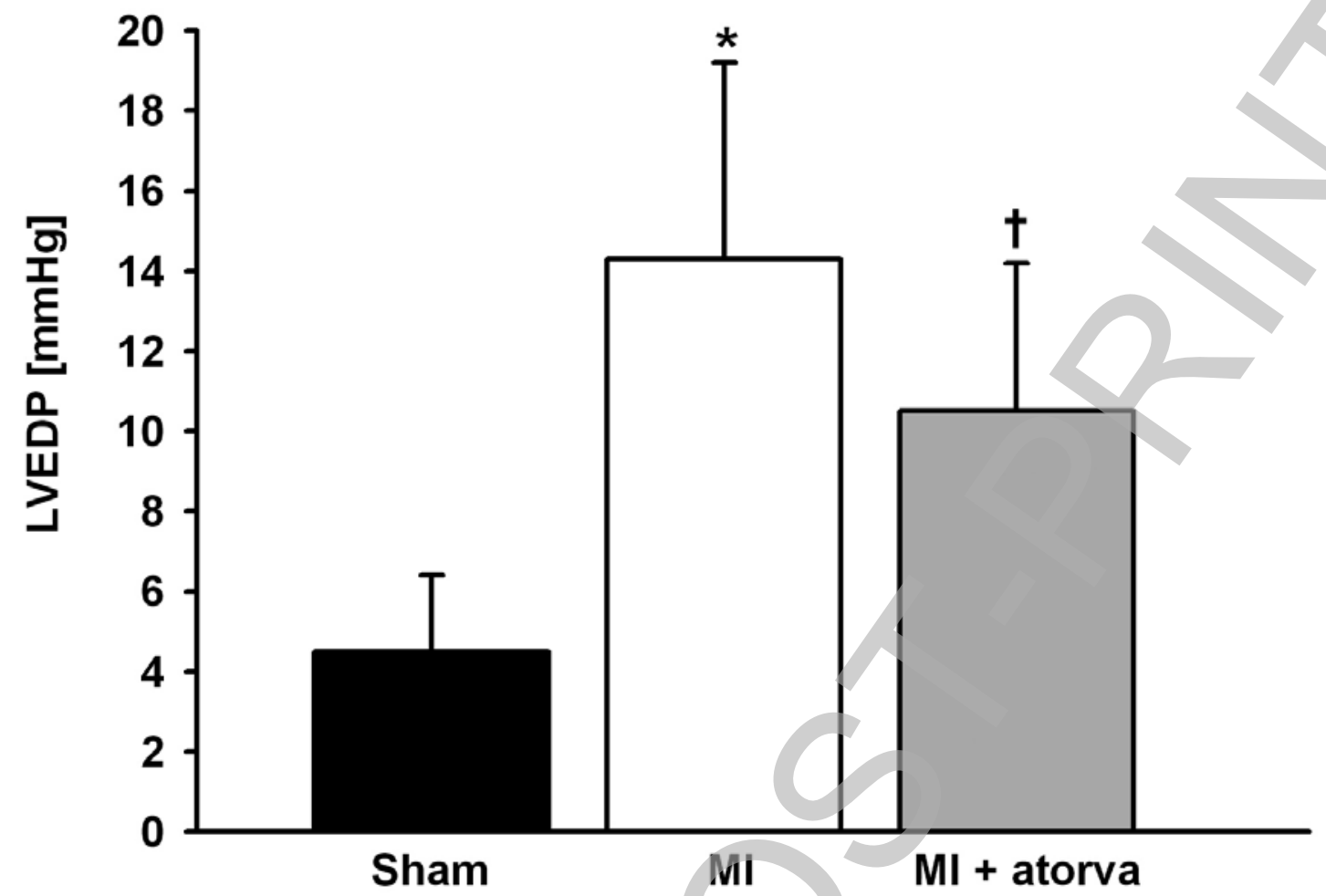

B

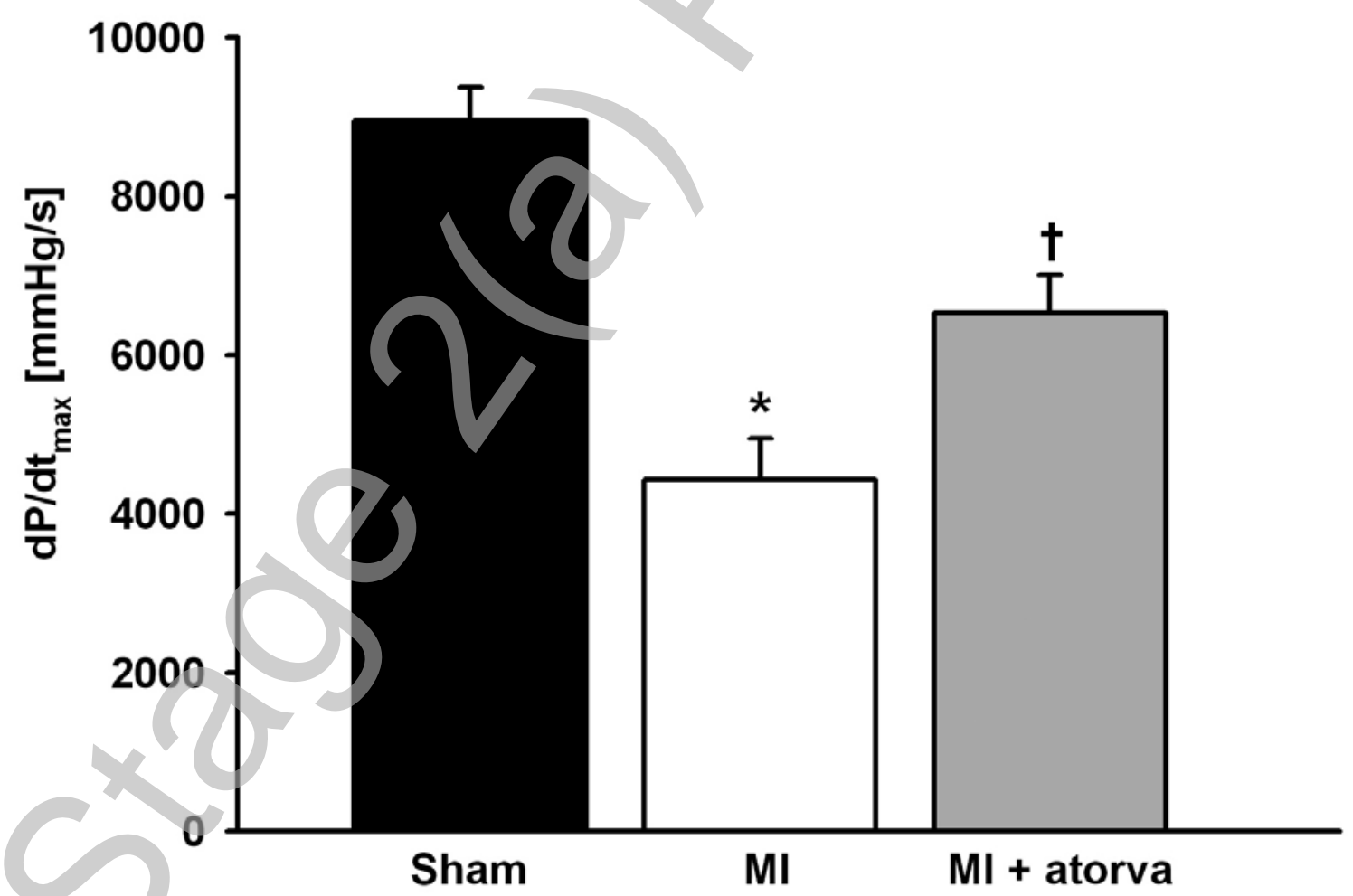

Figure 2 
A

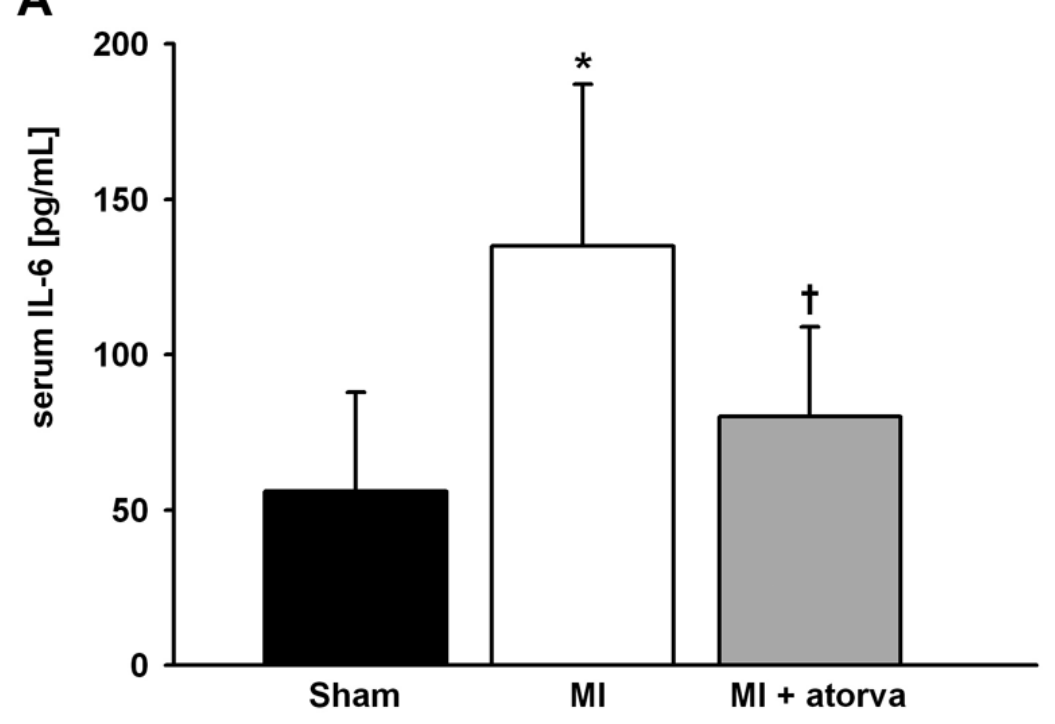

B

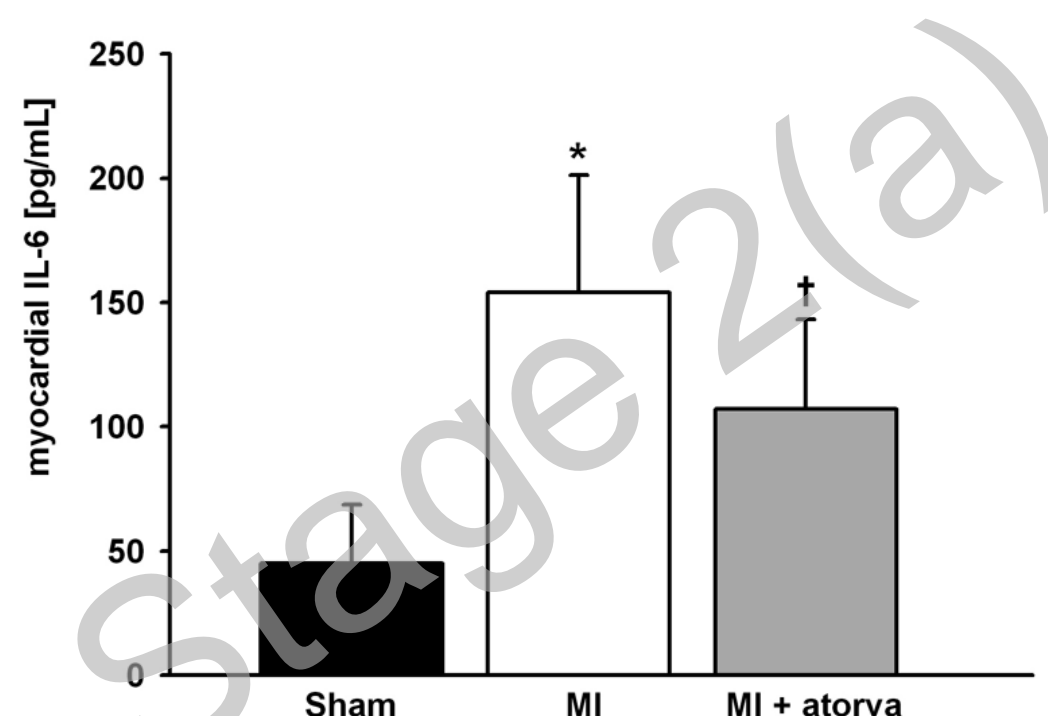

Figure 3
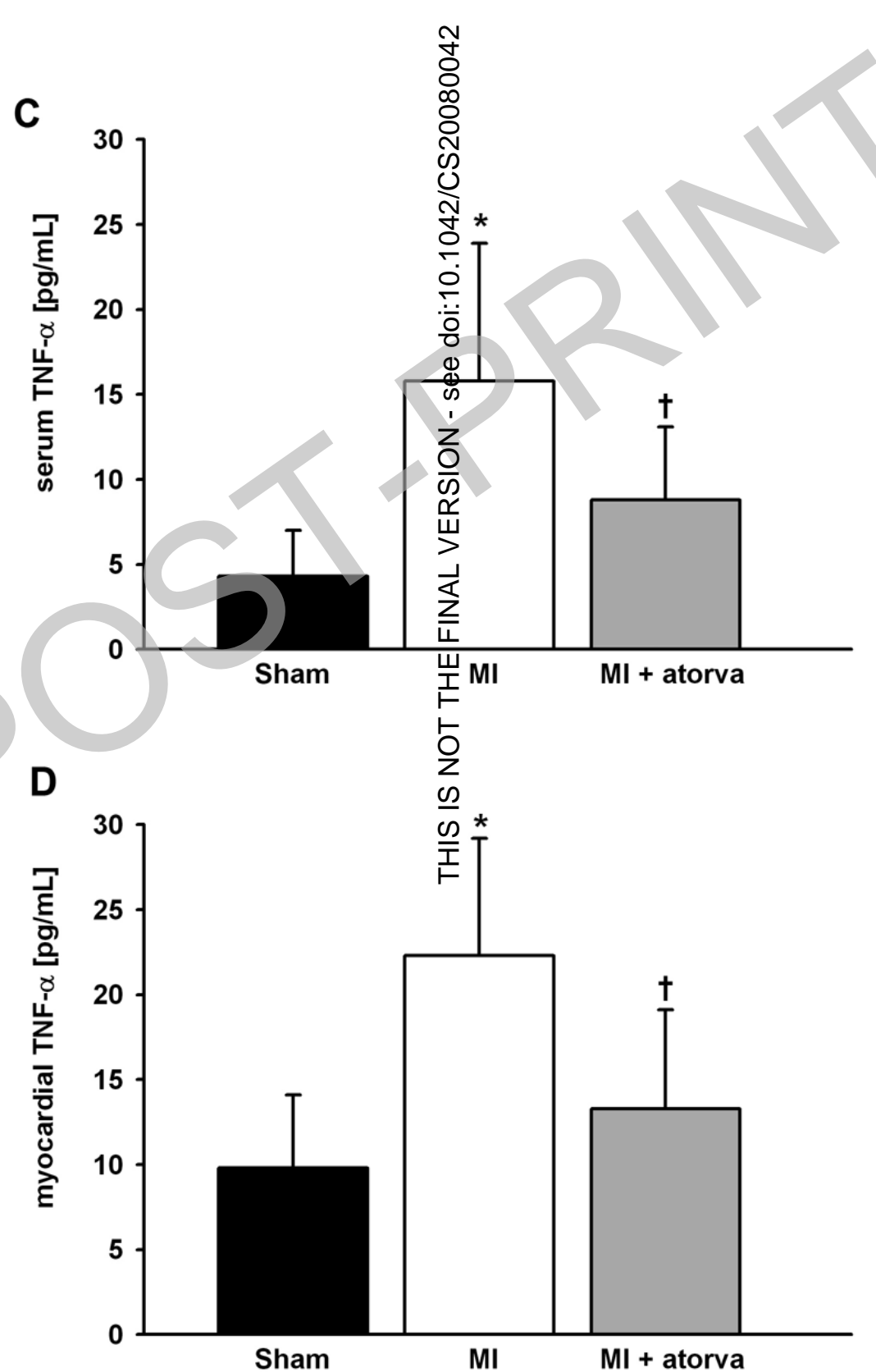

Licenced copy. Copying is not permitted, except with prior permission and as allowed by law. (c) 2008 The Authors Journal compilation (@ 2008 Biochemical Society 
A

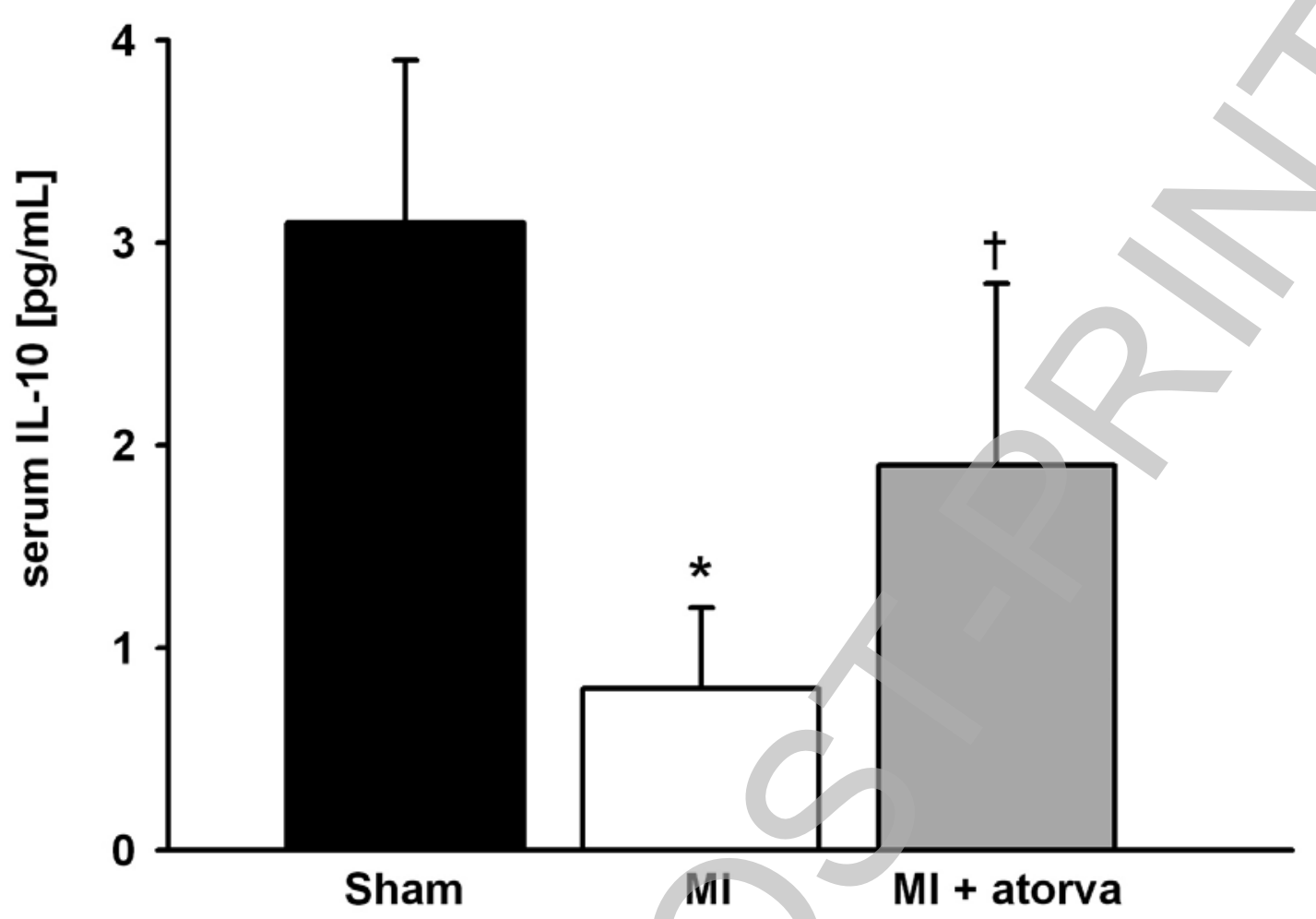

B

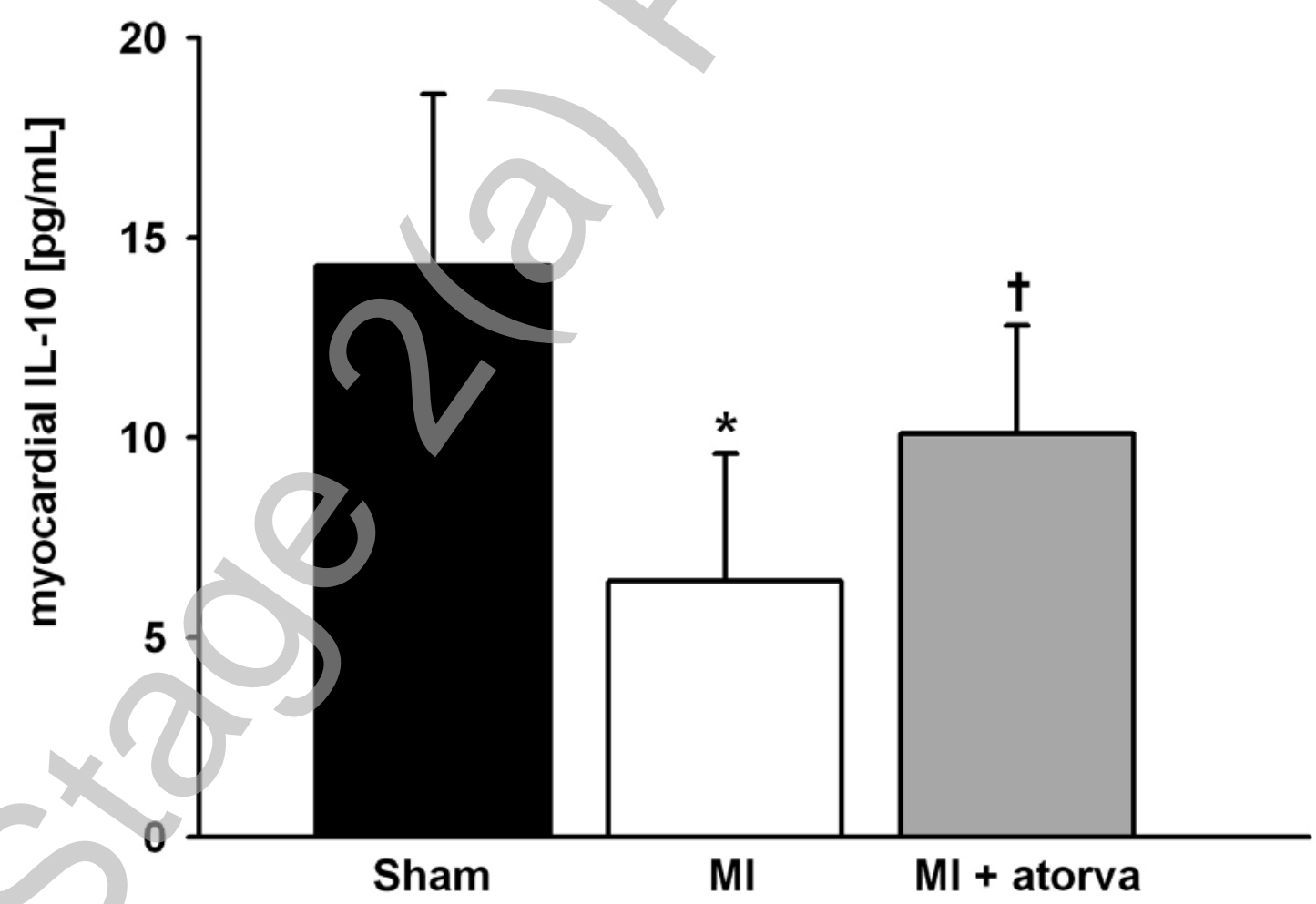

Figure 4 


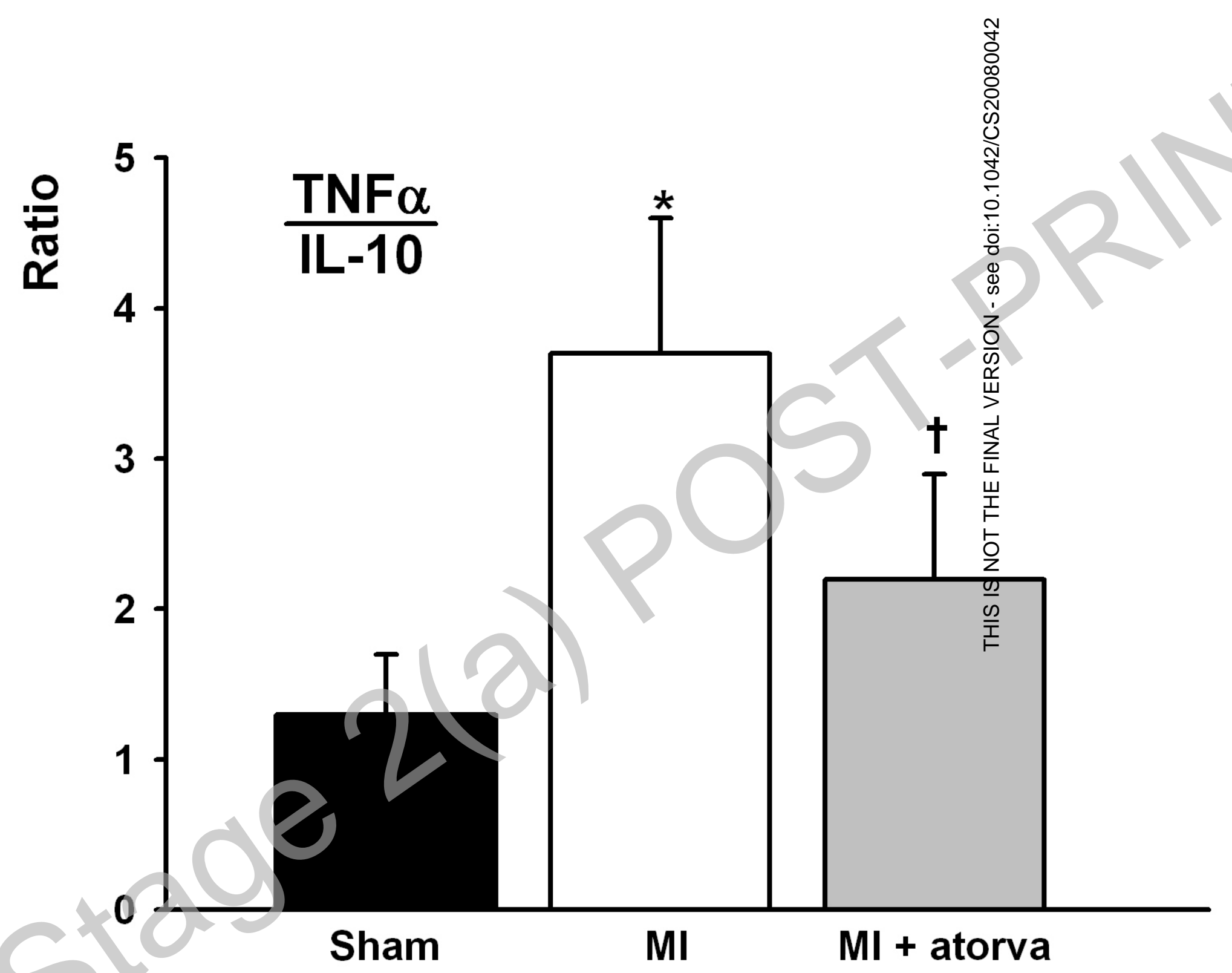

\section{Figure 5}


A

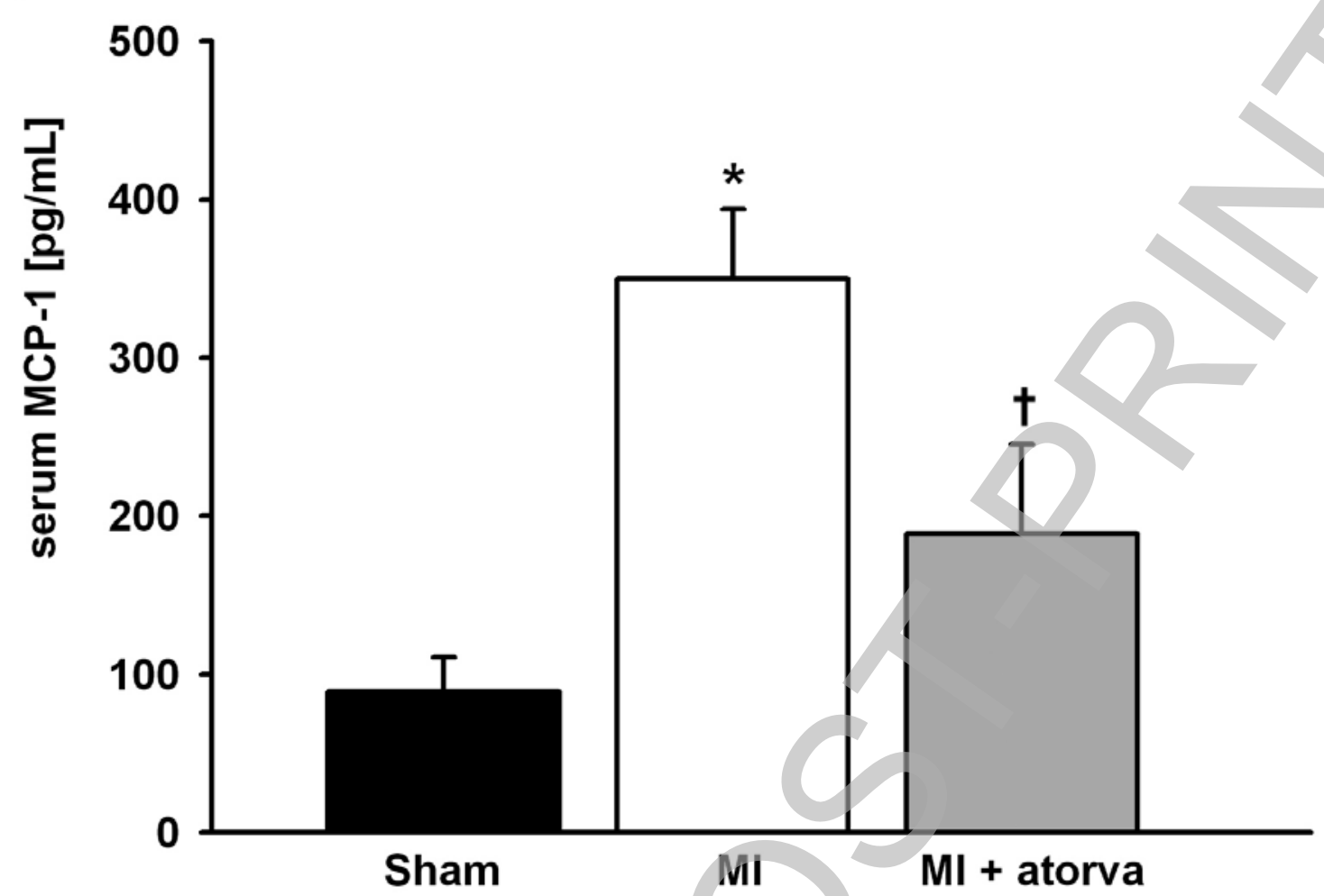

B

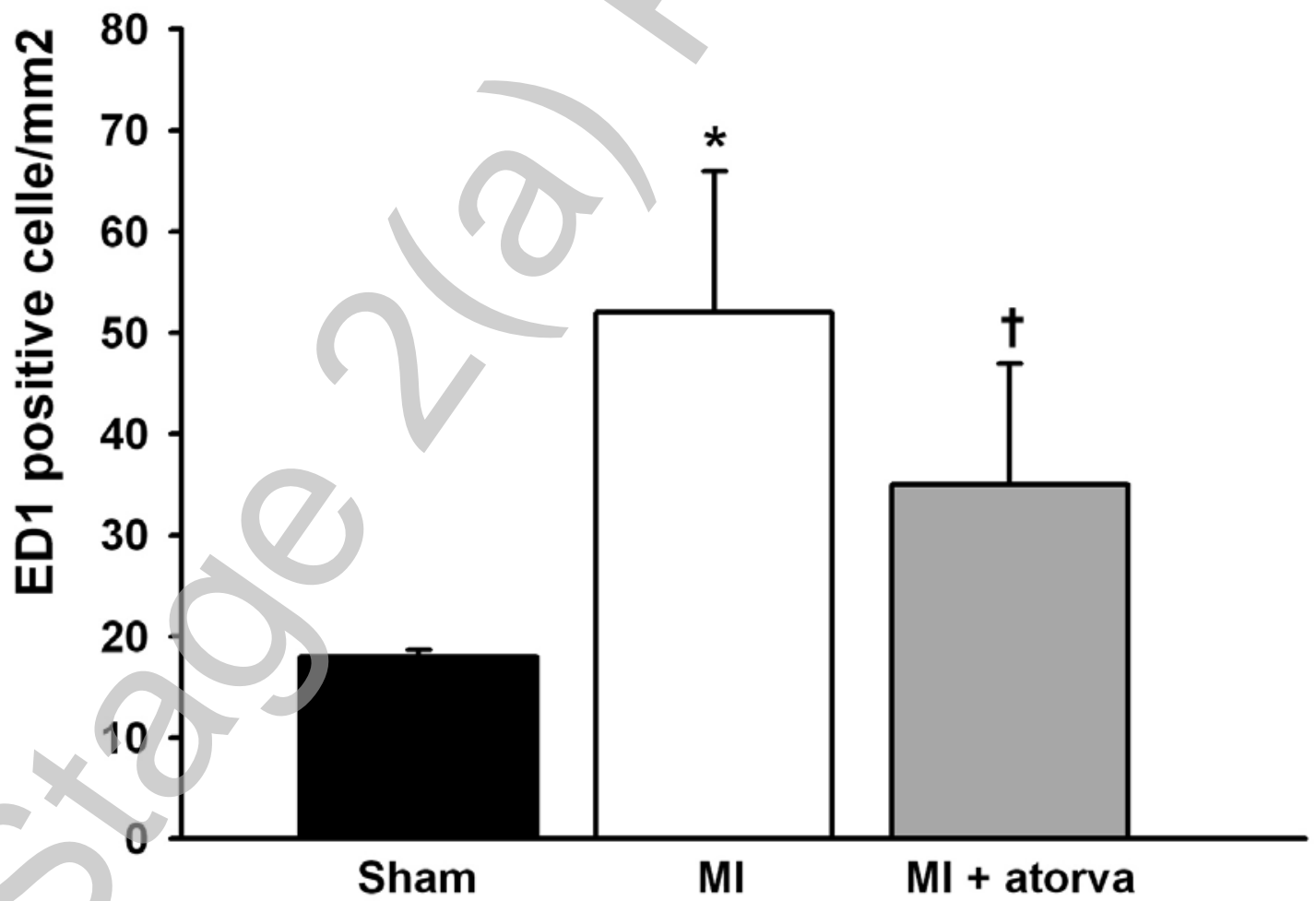

Figure 6 